OPEN ACCESS

Edited by:

Sasha Raoul Xola Dall,

University of Exeter, United Kingdom

Reviewed by:

Emma Doyle,

University of Exeter, United Kingdom

Ragen Trudelle-Schwarz McGowan,

Nestle Purina PetCare Company,

United States

*Correspondence:

Anindita Bhadra

abhadra@iiserkol.ac.in

Specialty section:

This article was submitted to

Behavioral and Evolutionary Ecology,

a section of the journal

Frontiers in Ecology and Evolution

Received: 23 July 2018 Accepted: 11 December 2018

Published: 21 December 2018

Citation:

Bhattacharjee D, Sau S and Bhadra A

(2018) Free-Ranging Dogs

Understand Human Intentions and

Adjust Their Behavioral Responses Accordingly. Front. Ecol. Evol. 6:232.

doi: 10.3389/fevo.2018.00232

\section{Free-Ranging Dogs Understand Human Intentions and Adjust Their Behavioral Responses Accordingly}

\author{
Debottam Bhattacharjee, Shubhra Sau and Anindita Bhadra*
}

Behaviour and Ecology Lab, Department of Biological Sciences, Indian Institute of Science Education and Research Kolkata, Mohanpur, India

Domestic dogs (Canis lupus familiaris) are remarkably sensitive and responsive while interacting with humans. Pet dogs are known to have social skills and abilities to display situation-specific responses, but there is lack of information regarding free-ranging dogs which constitute majority of the world's dog population. Free-ranging dogs found in most of the developing countries interact constantly with familiar and unfamiliar humans receiving both positive and negative behavior. Thus, understanding human intentions and subsequent behavioral adjustments are crucial for dogs that share habitats with humans. Here we subjected free-ranging dogs to different human social communicative cues (friendly and threatening - low and high), followed by a food provisioning phase, and tested their responsiveness. Dogs exhibited higher proximity seeking behavior as a reaction to friendly gesture whereas, they were prompted to maintain distance depending on the impact of the threatening cues. Interestingly, only the high-impact threatening had a persistent effect which also remained during the subsequent food provisioning phase. An elevated approach in the food provisioning phase elicited the dependency of freeranging dogs on humans for sustenance. Our findings suggest that free-ranging dogs demonstrate behavioral plasticity in interactions with humans; which provides significant insights into the establishment of the dog-human relationship on streets.

Keywords: free-ranging dogs, human intentions, communication, behavioral plasticity, dog-human relationship, threat perception

\section{INTRODUCTION}

Recent trends in research on interspecific interactions have unveiled several important aspects regarding the interplay of the component species. Investigating the eco-ethology of one component species and its trajectories can provide adequate information on the other (Bertness and Callaway, 1994; Thompson, 1999). Human-animal interaction is one such field that attracts researchers to find solutions for evolving problems like human-animal conflict, spread of zoonoses, uncontrolled population growth of unwanted species, etc. In the recent times, studies on human-animal interactions have enabled us to grasp evolutionary processes like domestication (Hare et al., 2002; Miklósi and Soproni, 2006). Such scientific investigations, coupled with comparative analyses, also help us understand the functionality of behaviors and communicative intents of species. As the first domesticated species, dogs have spent a considerably long period of time socially interacting with humans (Morey, 2006; Larson et al., 2012; Perri, 2016). Thus, exploring the dog-human interaction paradigm, is specifically helpful to analyse the underlying dynamics of the domestication process that enabled the transition of wolf-like ancestors to man's best friend. 
Domesticated dogs interact with humans regularly and possess social abilities to respond to various human actions (Hare and Tomasello, 2005; Miklósi and Soproni, 2006). Dogs are highly sensitive to human communicative cues like pointing, touching, body orientation etc. and in utilizing such cues to find hidden rewards such as food (Hare et al., 2002; Miklósi and Soproni, 2006). It has been suggested that domestication played a pivotal role in the development of human-like social skills in dogs (Hare and Tomasello, 2005). At the same time, substantial evidence for the importance of life history and ontogenic experience with humans in the development of the dog-human relationship is also present (Wynne et al., 2008; Dorey et al., 2010). Dogs have been shown to flexibly adjust their behavior in several interactive instances with humans such as, avoiding pointing cues provided by "unreliable humans" (Takaoka et al., 2015), following pointing cues only on being rewarded in a preceding trial, thereby showing the ability to adjust behavioral responses (Bhattacharjee et al., 2017a) etc. One study also confirmed dogs' understanding of human attentional states, suggesting that dogs can specifically ask for help when a human is paying attention to him/her (Miklósi et al., 2000). Dogs have been shown to focus on the human body and face while decoding intentions (Nagasawa et al., 2011). Vas et al. (2005) found that pet dogs can differentiate between friendly and threatening cues provided by an unfamiliar human and can display situation-relevant behavior. The same study also reported breed-specific differences of dogs' responsiveness due to varying levels of sensitivity toward humans. Except for one study mentioned above (Bhattacharjee et al., 2017b), all the other studies explored behavioral plasticity in pet dogs and hence are not representative of free-ranging dogs that are not under the direct supervision, and thereby influence, of humans.

Despite the fact that free-ranging dogs make up almost $80 \%$ of the world's dog population (Boitani and Ciucci, 1995; Hughes and Macdonald, 2013), studies on free-ranging dogs are greatly lacking. They are partially dependent on humans for their sustenance, but their activities are not directly controlled by humans (Sen Majumder et al., 2014; Bhadra et al., 2016; Paul et al., 2016). Unlike pet dogs, who are completely dependent on humans, the free-ranging subpopulation receive both positive and negative human interactions; humans play the most significant role in mortality of these dogs (Paul et al., 2016), while also being the primary provider of food. This particular difference, along with other ecological parameters pertaining to survival (competition for food, inter-group dynamics etc.) make free-ranging dogs somewhat different from pets. The interaction between free-ranging dogs and humans on the streets are quite complex and dynamic. They usually avoid human contact but can form strong bonds over repeated positive interactions with unfamiliar humans (Bhattacharjee et al., 2017b). Additionally, they need to understand and decipher human intentions clearly. A recent study showed that very young pups of freeranging dogs follow simple human pointing cues but no longer demonstrate this behavior when they grow up and start foraging. A greater risk of negative impact from humans like beating, harassment and threatening are probably the prime reasons for such plasticity in point-following behavior (Bhattacharjee et al., 2017a). However, this study does not provide us insights into the dogs' understanding of social cues that are used by humans in day to day interactions with these dogs.

Here we used three different types of commonly used human social communicative cues while interacting with dogs on streets. The cues differed in terms of their actions and representations. The friendly cue illustrated an affiliative gesture, while the low and high impact threatening cues had negative display of gestures. In addition to the three cues, we used a neutral cue as control. We tested the responses of the dogs to the cues and investigated the carry over effects in a post-cue food provision phase. We hypothesized that the dogs would react positively upon receiving the friendly cue showing higher proximity and approach behavior. Additionally, the dogs would avoid human proximity and adjust their responses in parallel with the level of threat perceived in the two threatening cues.

\section{MATERIALS AND METHODS Subjects and Study Area}

We tested 120 adult, physically fit (physically fit in appearance without any visible sign of injury or disease) solitary freeranging dogs. Dogs were located randomly in different areas of West Bengal, India (see Figure S1a) for the experimentKalyani $\left(22^{\circ} 58^{\prime} 30^{\prime \prime} \mathrm{N}, \quad 88^{\circ} 26^{\prime} 04^{\prime \prime} \mathrm{E}, \quad\right.$ Figure S1b), Kolkata $\left(22^{\circ} 57^{\prime} 26^{\prime \prime} \mathrm{N}, 88^{\circ} 36^{\prime} 39^{\prime \prime} \mathrm{E}\right.$, Figure S1c), Mohanpur $\left(22^{\circ} 56^{\prime} 49^{\prime \prime} \mathrm{N}\right.$ and $88^{\circ} 32^{\prime} 4^{\prime \prime} \mathrm{E}$, Figure S1d) and Sodepur $\left(22^{\circ} 69^{\prime} 82^{\prime \prime} \mathrm{N}\right.$ and $88^{\circ} 38^{\prime} 95^{\prime \prime} \mathrm{E}$, Figure S1e). We avoided testing groups of dogs in this study. All dogs were sexed and their phenotypic details such as coat color, scar marks were recorded so as to prevent resampling. To further rule out any possibility of resampling, we tested dogs from different locations on different days.

\section{Experimental Procedure}

We used four different experimental conditions incorporating targeted social cues to investigate the response of solitary freeranging dogs toward an unfamiliar human. We tested separate sets of 30 dogs in each of the four experimental conditions. All the experimental trials were conducted on the same locations where the focal free-ranging dog was found (e.g., streets, markets, residential areas etc.). Assignment of experimental conditions was performed in a random fashion. One piece of raw chicken $(10-12 \mathrm{~g})$ was used as food.

The experimenters (E1 and E2) were consistent throughout the study and played specific roles. Both E1 and E2 were male, 27 years old, 160-165 cm in height and slim, thus of similar physical build. Video recording was done from a distance using a Sony HDR-PJ410 camera mounted on a tripod.

The experimental conditions comprised of five phases, carried out in the following order (Figure S2).

\section{Attention Seeking Phase}

E2 attracted the attention of a solitary dog using very short vocalizations for 1-2 s (Bhattacharjee et al., 2017a). This step was necessary as we found some dogs lying down, resting or dozing. To keep the protocol consistent, E2 carried out this step in all the four experimental conditions. 


\section{Transition Phase}

Once the dog was alerted, E2 immediately left the place and stood behind the camera, which was kept at a minimum distance of $4.5 \mathrm{~m}$ from the dog. E1 arrived near the position where E2 was initially standing. This whole procedure was completed within 10 s. E2 shifted the camera whenever a dog moved to keep the minimum distance of $4.5 \mathrm{~m}$.

\section{Social Cue Phase/SCP}

E1 stood approximately $1.5 \mathrm{~m}$ away from the dog, facing it. Since the dogs were free-ranging and not on leash, E1 had to adjust his position in order to maintain the approximate distance of $1.5 \mathrm{~m}$. After standing at the specified spot, E1 provided any of the predetermined social cue for $30 \mathrm{~s}$ :

- Friendly Cue (FC)-E1 displayed a positive gesture by bending slightly forward and extending both the arms (see Movie S1). Though such posture has been described as a threat in the existing canine literature, people in India typically use such gestures (sometimes paired with positive vocalizations) to provide positive social rewards to dogs (Figure S3). E1, while providing the social cue, gazed and tried to maintain eye-contact with the dog. E1 did not touch the focal dog deliberately in order to avoid any potential bias of social contact.

- Low impact threatening (LIT)-E1 raised one of his hands (counterbalanced), kept it motionless and gazed at the dogs (see Movie S2). The cue differed from FC in having a negative display of human gesture. People on the streets often raise one of their hands to scare, threaten or shoo away dogs. We have adopted the same gesture in our protocol to investigate the effects and associated responses.

- High impact threatening (HIT)-This phase differed from LIT in terms of impact. Here E1 used a $0.45 \mathrm{~m}$ long solid wooden stick in his hand (counterbalanced) while providing the gesture and directly facing the dogs (see Movie S3). E1 had to hide the wooden stick in the transition phase before enacting the gesture.

- Neutral Cue (NC)-Here, E1 stood in a neutral posture and looked straight ahead and did not enact any gesture.

\section{Food Transfer Phase}

Immediately after SCP, food was provisioned. E2 came quickly, handed over the food to E1 and went back to his position behind the camera. The process was completed within $10 \mathrm{~s}$ and care was taken to ensure that the focal dog did not see the transfer of food to E1. Moreover, both E1 and E2 stood opposite to the focal dog and did not face him/her during the food transfer.

\section{Food Provisioning Phase/FPP}

E1 again adjusted his position to keep the distance consistent in case a focal dog moved and placed the food on the ground. The food was placed at a distance of $0.3 \mathrm{~m}$ from E1, thus at a distance of $1.2 \mathrm{~m}$ from the dog. E1 stood in a neutral position after placing the food and looked straight ahead, without making eye contact with the dog (see Movie S4). FPP lasted 30 s or until the dog obtained the food, whichever was earlier. Food was removed in case a dog did not obtain it.
Except for the SCP, all the other phases were constant and identical across the experimental conditions.

\section{Data Analysis and Statistics}

We coded all the important behaviors relating to the experiment, which have been listed in the ethogram below (Table 1).

Shapiro -Wilk tests were used to check for normality of the data. We found the data to be not normally distributed, thus non-parametric tests were carried out. Generalized linear models (GLM) were performed using "Ime4" package of R Studio. AIC values were compared in order to get the best-fitting models. A second coder naïve to the purpose of the study coded $20 \%$ of the data to check inter-rater reliability. It was perfect for number of approach (cohen's kappa $=1.00$ ), almost perfect for duration of proximity (cohen's kappa $=0.93$ ), gazing (cohen's kappa $=0.90)$, and latency (cohen's kappa $=0.96$ ). The alpha level was 0.05 throughout the analysis. Post-hoc comparisons were done with Bonferroni correction method whenever required. Coding of videos was done using Solomon Coder version 17.03.22. Statistical analyses were performed using R (R Development Core Team, 2015) and StatistiXL version 1.11.0.0.

\section{RESULTS}

Various statistical tests were carried out for the analysis. Since we have only compared the parameters of SCP and FPP in all possible combinations, description of some of the post-hoc statistical tests have been presented in the Supplementary Material to avoid congestion in the main text.

\section{Number Approached}

Dogs approached differently in SCP and FPP of the four conditions (Contingency $\chi^{2}: \chi^{2}=10.439$, df $=3, p=0.015$, Figure 1). In the NC condition, initially 4 individuals approached, while the number increased to 17 in FPP, the change being statistically significant (Goodness of fit $\chi^{2}$ : $\left.\chi^{2}=8.048, \mathrm{df}=1, p=0.005\right)$, thereby indicating a distinct positive impact of food. However, we did not find any difference between the two phases in the FC condition as dogs equally responded to both positive gestures (25) and food (30), more than expected by chance alone (Goodness of fit $\chi^{2}: \chi^{2}=0.455$, $\mathrm{df}=1, p=0.50)$. The LIT condition had a very momentary impact as only 1 individual approached in SCP, while 13 individuals approached in FPP (Goodness of fit $\chi^{2}: \chi^{2}=10.246$, $\mathrm{df}=1, p=0.001)$. Thus, dogs flexibly adjusted their behavior and tended to approach more when food was provided. Unlike the LIT condition, we found a strong effect of HIT, where none of the individuals approached initially and only 1 (Goodness of fit $\chi^{2}: \chi^{2}=1.000, \mathrm{df}=1, p=0.317$ ) in the later phase, when the food reward was offered. This was suggestive of the dogs' perception of human intentions based on an immediate encounter.

We compared the number of approaches across conditions for both SCP and FPP. Significantly higher number of dogs approached in SCP in response to FC, as compared to the NC, LIT, and HIT conditions (see Table S1). We noticed a marginal difference between the NC and HIT conditions (see Table S1). 
TABLE 1 | List of behaviors coded from the videos and their definitions.

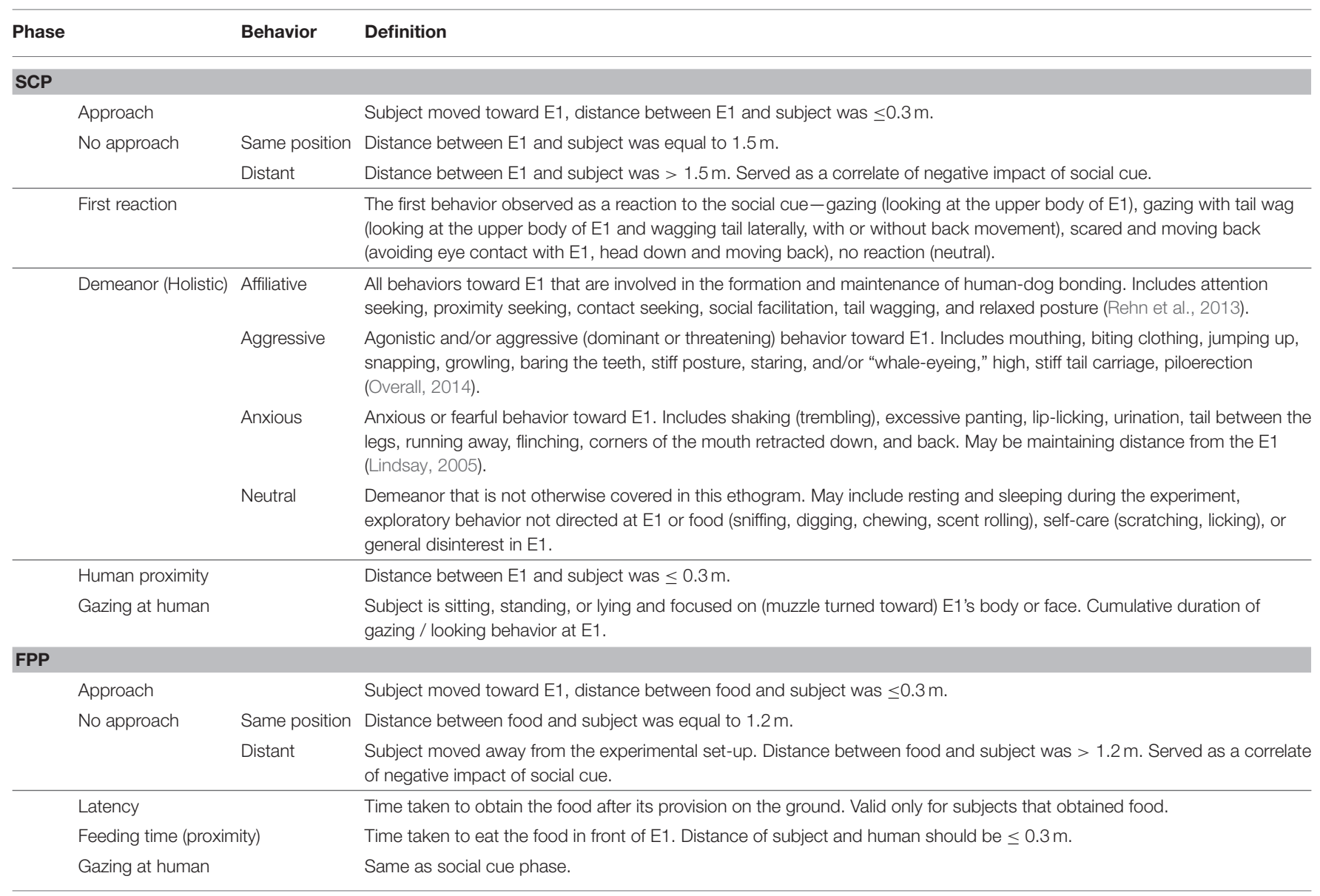

Additionally, the number of approaches in the SCP of LIT did not differ from NC and HIT conditions (see Table S1).

We compared the number of approaches among FPP of the four conditions. Dogs approached significantly more in FC compared to LIT (Goodness of fit $\chi^{2}: \chi^{2}=6.721$, $\mathrm{df}=1$, $\mathrm{p}=0.010$ ) and HIT (Goodness of fit $\chi^{2}: \chi^{2}=27.129$, $\mathrm{df}=1$, $p<0.0001$ ), but not NC (Goodness of fit $\chi^{2}: \chi^{2}=3.596, \mathrm{df}=1$, $p=0.058)$, again implying the role of the food provisioned. In the HIT condition, the number of dogs that approached was significantly lower than the LIT condition (Goodness of fit $\chi^{2}$ : $\left.\chi^{2}=10.286, \mathrm{df}=1, p=0.001\right)$. In addition, we also found that the HIT cue differed from NC, but the number of approaches in FPP of LIT and NC conditions were comparable (see Table S1).

\section{No Approach}

Consistent with our hypothesis, we could not see any dog running or moving away in the NC and FC conditions, thereby dogs exclusively showed no approach of "same position" subcategory. Thus, we analyzed the data only from LIT and HIT conditions. We used the percentage of responses out of the total "no approach" cases for all the comparisons.

52 and $24 \%$ of the dogs were distant in SCP and FPP, respectively, in the LIT condition (Goodness of fit $\chi^{2}$ : $\chi^{2}=10.316, \mathrm{df}=1, p=0.001$, Figure 2). Consistent with this, we also found dogs showing significantly more distant positions in SCP $(73 \%)$ than the FPP $(45 \%)$ of the HIT condition (Goodness of fit $\chi^{2}: \chi^{2}=6.644, \mathrm{df}=1, p=0.01$, Figure 2). Further comparisons revealed a significant difference between the FPP of LIT and HIT conditions (Goodness of fit $\chi^{2}: \chi^{2}=6.391, \mathrm{df}=1$, $p=0.011$, Figure 2), where higher numbers of dogs stayed at the "distant" position in the HIT condition. However, we did not find any difference between the SCP of the two conditions (Goodness of fit $\left.\chi^{2}: \chi^{2}=3.528, \mathrm{df}=1, p=0.060\right)$.

\section{First Reaction to a Social Cue}

Quantification of the first reaction was important in terms of impact and effect of the social cues. We found that the reactions (see Table 1) were distributed differently in the four experimental conditions. In the NC condition, dogs showed varying levels of reactions. $60 \%$ of the dogs showed gazing behavior, $10 \%$ showed gazing with tail wagging, $30 \%$ stayed neutral, and displayed no particular reaction. None of the dogs showed a fear response. We found a significant difference among the proportion of individuals showing the different reactions (Goodness of fit $\chi^{2}$ : $\chi^{2}=25.200$, df $=3, p<0.0001$, Figure 3A). Gazing and no reaction were comparable and displayed at a higher rate than 


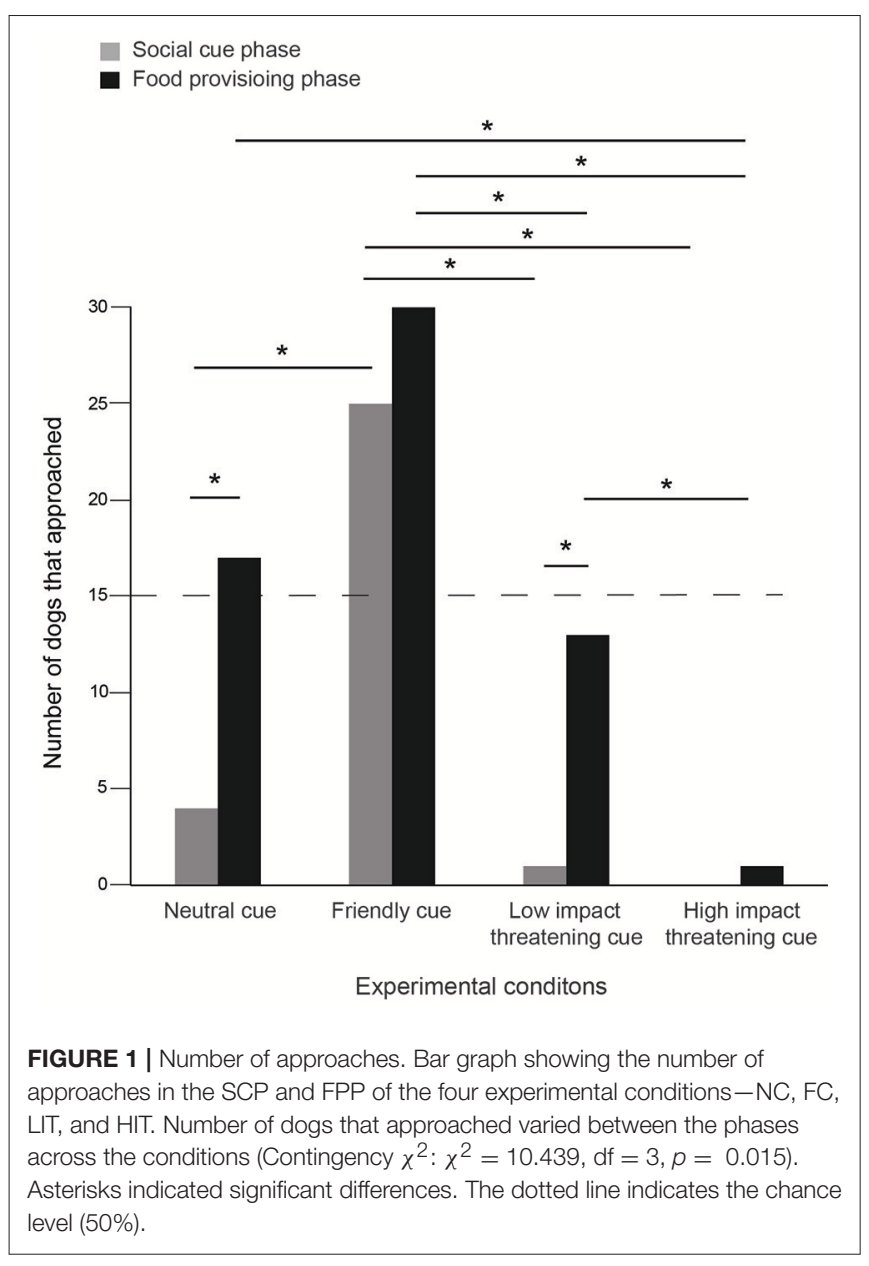

other behaviors (see Table S1). In the FC condition, we found $80 \%$ of the dogs showing gazing with tail wagging as their first reaction, while $20 \%$ showed gazing behavior only. No dog showed a fear response, and all dogs responded. Gazing with tail wagging occurred at a significantly higher rate than only gazing behavior (Goodness of fit $\chi^{2}: \chi^{2}=10.800$, df $=1, p=0.001$, Figure 3B). Except for a single individual, all the dogs reacted in the LIT condition. Sixty percent of the dogs showed fear response to the social cue at a significantly higher rate than both gazing (20\%) and gazing with tail wagging (17\%) behaviors (Gazing-Goodness of fit $\chi^{2}: \chi^{2}=6.000$, df $=1, p=0.014$; Gazing with tail wagging-Goodness of fit $\chi^{2}: \chi^{2}=7.348$, $\mathrm{df}=1, p=0.007$, Figure $3 \mathrm{C}$ ). In the HIT condition, $97 \%$ of the dogs showed fear response when the threatening gesture was enacted, whereas only one individual displayed gazing with tail wagging (Goodness of fit $\chi^{2}: \chi^{2}=26.133$, df $=1, p<0.0001$, Figure 3D).

\section{Demeanor}

Dogs displayed mostly neutral (43\%) and anxious (43\%) behaviors in the NC condition. Affiliative behaviors were shown at a lower rate than both the neutral and anxious behaviors (Goodness of fit $\chi^{2}: \chi^{2}=4.765$, df $=1, p=0.029$, Figure 4).

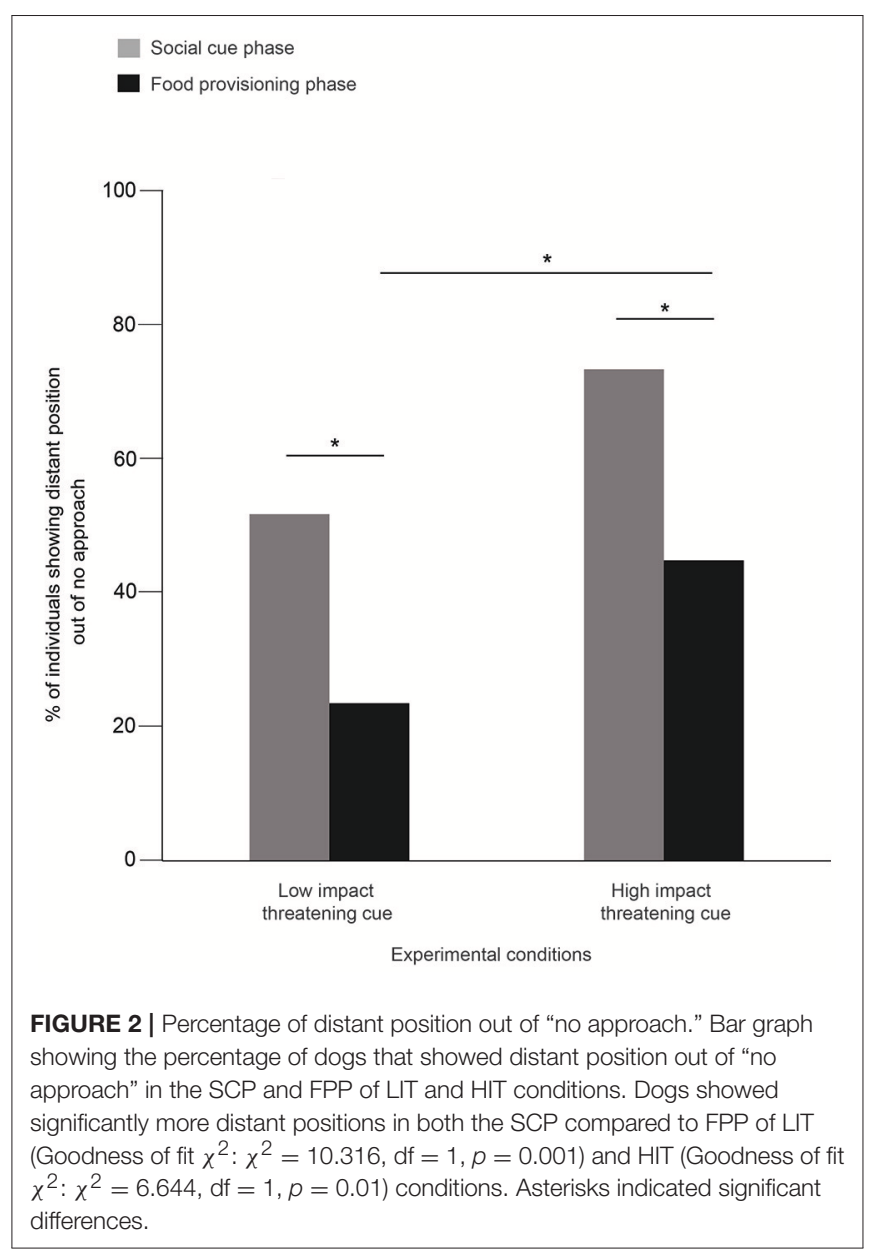

Agonistic or aggressive behaviors were absent. Unlike the outcomes in NC, majority of dogs (80\%) showed affiliative behaviors, rather than neutral (Goodness of fit $\chi^{2}: \chi^{2}=12.448$, $\mathrm{df}=1, p<0.0001$ ) and anxious behaviors (Goodness of fit $\chi^{2}$ : $\left.\chi^{2}=21.160, \mathrm{df}=1, p<0.0001\right)$ in FC. Aggression was not observed. In the LIT condition, $57 \%$ of the dogs showed anxious behaviors, which was higher than all the other three categories - (Neutral-Goodness of fit $\chi^{2}: \chi^{2}=6.545$, df $=1, p=0.011$; Affiliative-Goodness of fit $\chi^{2}: \chi^{2}=6.545$, df $=1, p=0.011$; Aggressive-Goodness of fit $\chi^{2}: \chi^{2}=9.800$, df $=1, p=0.002$ ). $97 \%$ of the dogs showed anxious behaviors in HIT condition. However, we did not see a statistical difference between the levels of anxious behavior shown in LIT and HIT conditions (Goodness of fit $\left.\chi^{2}: \chi^{2}=3.130, \mathrm{df}=1, p<0.07\right)$.

\section{Human Proximity}

Dogs showed varying levels of proximity to the human experimenter in the different conditions (Kruskal-Wallis test, $\chi^{2}=77.127$, df $=3, p<0.0001$, Figure 5). Post-hoc pairwise comparisons revealed that the duration of human proximity was higher in the FC condition compared to others (see Table S1). However, we did not find any difference between the duration of human proximity in the NC, LIT, and HIT conditions (see 


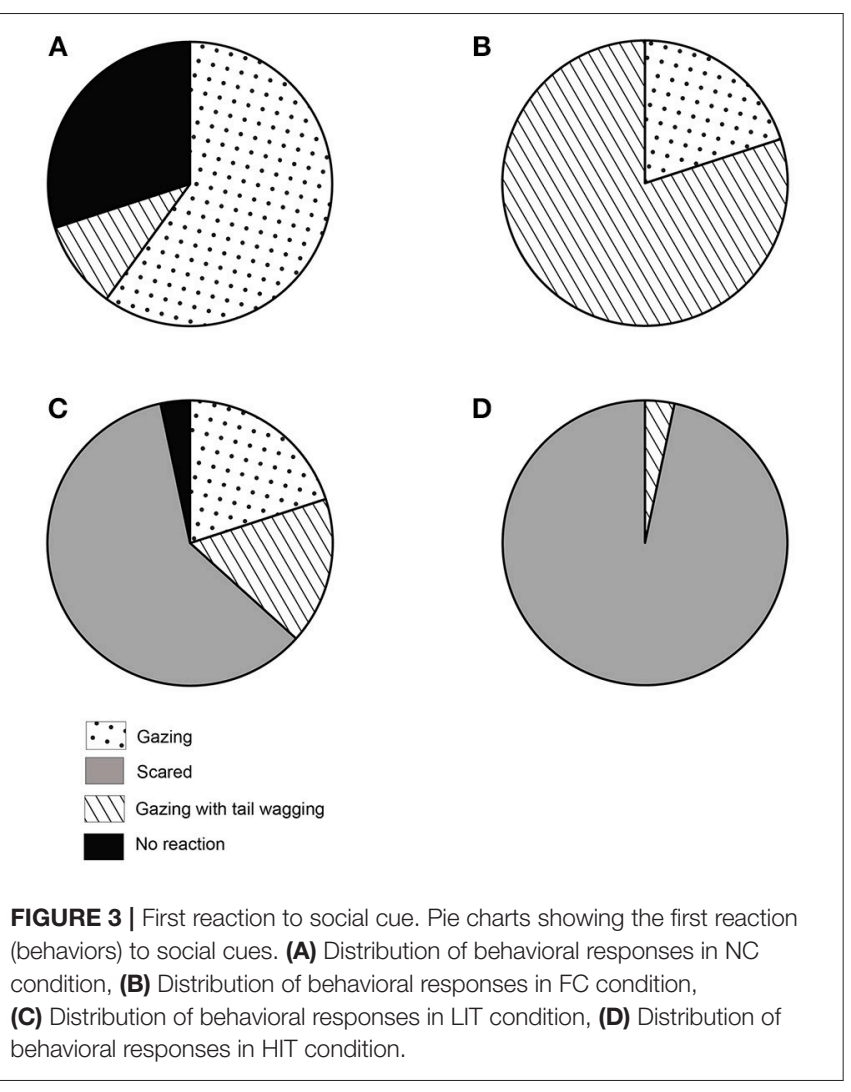

Table S1), indicating a general avoidance of human proximity in free-ranging dogs.

\section{Gazing}

GLM analysis revealed that both the LIT and the HIT conditions are significant predictors of gazing at E1 in the SCP (Figure 6, Table 2).

Interestingly, in the FPP, we found all the different conditions to be significantly contributing to the prediction of the duration of gazing behavior (Figure 6, Table 3). Dogs gazed the least $(0.46$ $\pm 1.69 \mathrm{sec}$ ) in the FPP of the FC condition.

\section{Latency and Feeding Time (Food Provision Phase Only)}

Individuals who approached the food, were considered for the latency comparisons $(N=60)$. We excluded the HIT condition from the analysis as only one dog approached and obtained the food reward. Individuals showed different latencies in the three conditions while approaching for the food (Kruskal-Wallis test, $\chi^{2}=34.011, \mathrm{df}=2, p<0.0001$, see Figure S4). In the FC condition, the dogs approached faster than the NC (MannWhitney $U$-test, $\mathrm{U}=452.000$, df $1=17, \mathrm{df} 2=30, p<0.0001)$ and LIT (Mann - Whitney $U$-test, $U=374.000$, df1 $=30$, df $2=13$, $p<0.0001)$ conditions. Latencies were comparable in the $\mathrm{NC}$ and LIT conditions (Mann - Whitney $U$-test, $U=156.500$, df1 $=17$, df2 $=13, p=0.053)$.

We found one individual in the FC condition that approached but did not obtain the reward. Thus, we removed the data point

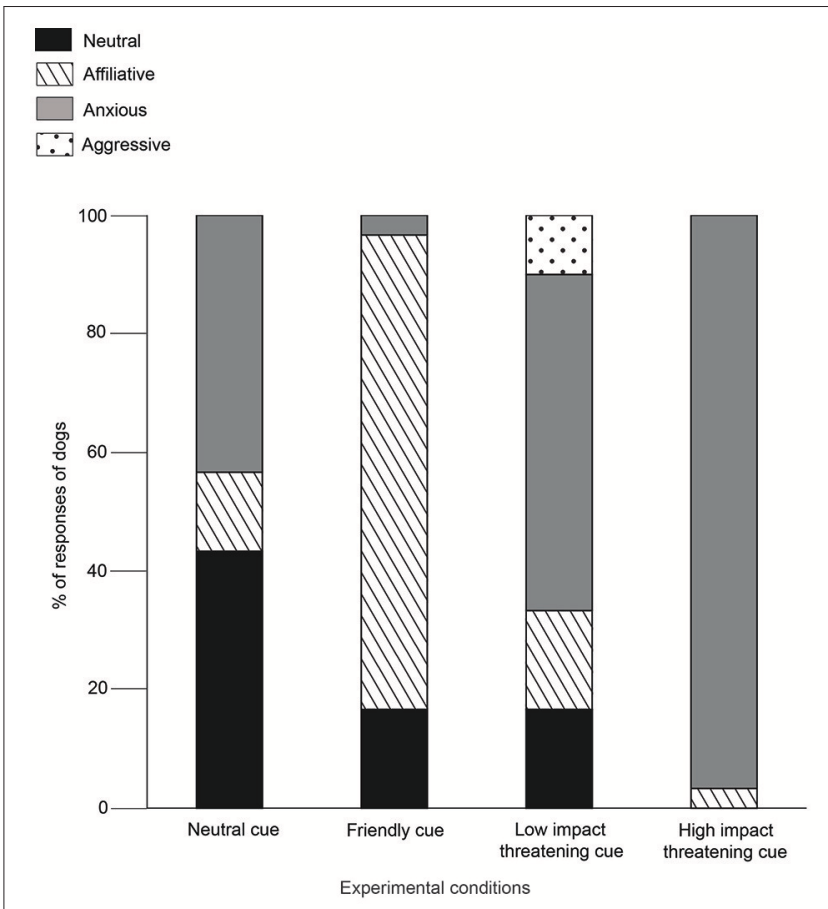

FIGURE 4 | Demeanours (Holistic). Stacked bar graph showing the demeanours of dogs in SCP of NC, FC, LIT, and HIT conditions.

for the analysis of feeding time $(N=59)$. We found a significant difference in feeding time (Kruskal-Wallis test, $\chi^{2}=8.366$, $\mathrm{df}=2, p=0.015$, see Figure S5). Post-hoc pairwise comparisons further revealed a significant difference between feeding times of FC and LIT conditions (Mann-Whitney $U$-test, $U=298.000$, $\mathrm{df} 1=29, \mathrm{df} 2=13, p=0.002)$. We found a relatively short feeding time in the LIT condition $(2.77 \pm 0.72 \mathrm{~s})$ compared to the FC $(4.58 \pm 2.02 \mathrm{~s})$. Moreover, we did not see any difference between the other two comparisons (see Table S1).

\section{DISCUSSION}

Our results underline free-ranging dogs' behavioral plasticity in the context of interactions with unfamiliar humans. Dogs adjusted their behavior and showed situation-relevant response to the social cues. Overall, they exhibited a tendency to approach more when food was provisioned compared to the social cue phases, emphasizing the dependence on humans for sustenance. However, comparable but higher levels of approach in the FC condition identified an important role of positive social actions from humans, in order to encourage the initiation of an affiliative relationship. The comparatively higher duration of human proximity in the FC further strengthens this statement. The influence of low-impact threatening cues was very momentary while the effect of the high-impact threatening cues remained even when food was provided. Moreover, dogs avoided the unfamiliar human (E1) with a comparatively higher distance in HIT compared to LIT. Thus, dogs were able to distinguish between the impacts of the threatening cues and responded 


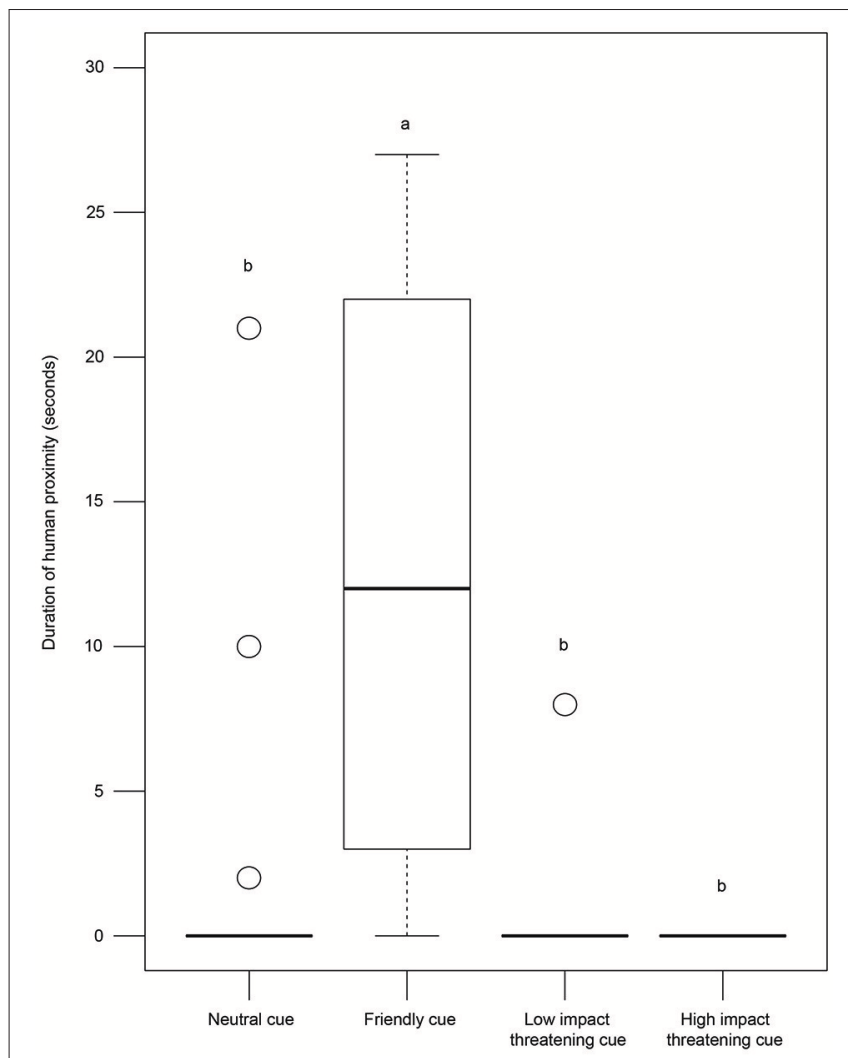

FIGURE 5 | Duration of human proximity. Box and whisker plot illustrating the duration of human proximity of dogs in SCP of NC, FC, LIT, and HIT conditions. Dogs showed significantly higher proximity to E1 in the FC (12.43 $\pm 9.54 \mathrm{sec}$ ) than in other conditions. Boxes represent interquartile range, horizontal bars within boxes indicate median values, and whiskers represent the upper range of the data. "a" and "b" indicate significant differences.

accordingly, illustrating an optimized strategy. Additionally, the initial reactions and demeanors were consistent with the differential approach of dogs to the corresponding cues.

Apart from showing affiliative responses in the FC condition, dogs showed adjustments and plasticity in their anxious or fearful responses during the threatening cue conditions. Gazing behavior in SCP was predicted by LIT and HIT conditions as dogs gazed more, probably indicating their hesitant nature to approach and also gauging human intentions. On the other hand, FC, LIT, and HIT conditions predicted the gazing response in FPP. It is important to note that the short duration of gazing at E1 in the FC condition could be the linked to dogs' certainty due to affiliative human action. This was also supported by a significantly faster approach to the food. Moreover, dogs depicted a tendency to spend more time while feeding in the FC condition compared to LIT. Thus, the free-ranging dogs acted very specifically in the different conditions displaying a range of social responses that had a high degree of parity with the social cue provided in the experiment.

Free-ranging dogs live in human dominated environments and heavily depend on humans for food (Bhadra and Bhadra, 2014; Bhadra et al., 2016). In addition to scavenging, they directly

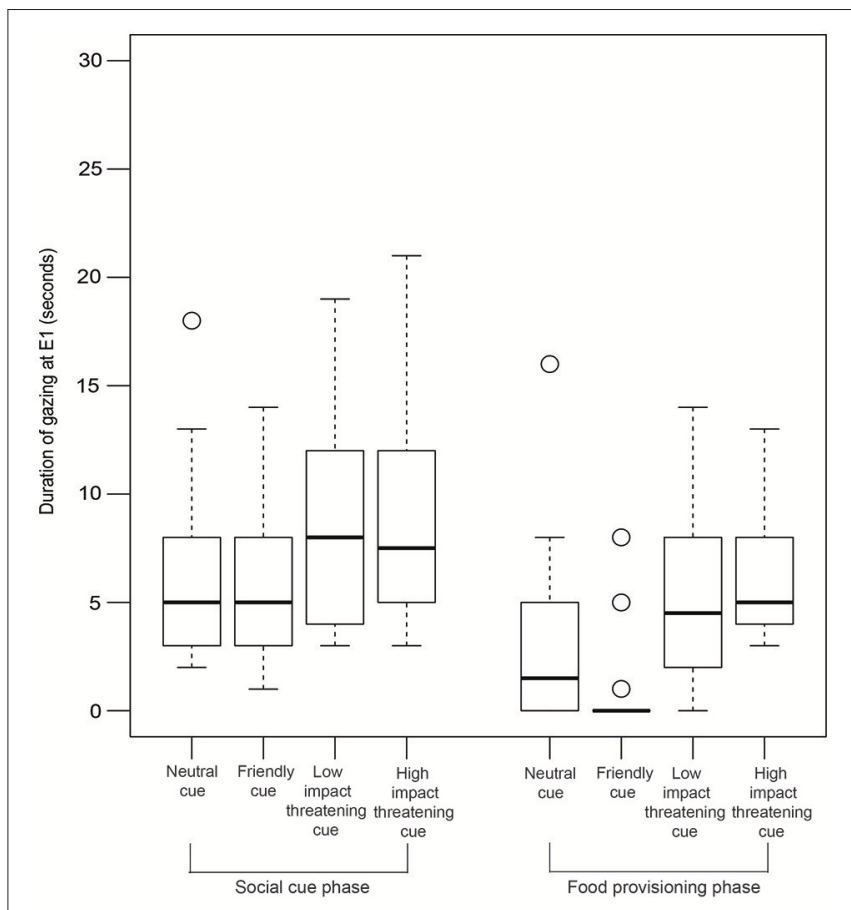

FIGURE 6 | Duration of gazing. Box and whiskers plot showing the duration of gazing at E1 in SCP and FPP of NC, FC, LIT, and HIT conditions. Boxes represent interquartile range, horizontal bars within boxes indicate median values, and whiskers represent the upper range of the data.

TABLE 2 | GLM results showing the effect of experimental conditions on gazing behavior in the SCP.

\begin{tabular}{lcccc}
\hline & Estimate & Standard error & z-value & $\operatorname{Pr}(>|\mathbf{z}|)$ \\
\hline \multicolumn{2}{l}{ FIXED EFFECTS } & & & \\
Intercept & 1.75786 & 0.07581 & 23.188 & $<2 \mathrm{e}-16^{\text {***}}$ \\
Condition FC & 0.01143 & 0.10691 & 0.107 & 0.914865 \\
Condition HIT & 0.43937 & 0.09722 & 4.520 & $6.2 \mathrm{e}-06^{* \star *}$ \\
Condition LIT & 0.37828 & 0.09841 & 3.844 & $0.000121^{* \star *}$ \\
\hline
\end{tabular}

${ }^{\star \star \star} P \leq 0.001$.

beg for food from humans (Bhadra and Bhadra, 2014; Sen Majumder et al., 2014). However, getting or retrieving food items can lead to consequences like beating and harassment, which probably have made these dogs opportunistic foragers. In order to avoid negative human impact and maximize the success of getting food, dogs need to identify reliable humans. General avoidance of direct physical contact with unfamiliar humans (Bhattacharjee et al., 2017b) may be a process involved in the same strategy. However, the flexibility might have been achieved by dogs upon receiving positive human reinforcements. For example, adult free-ranging dogs have earlier been shown to adjust their point-following behavior based on reliability of unfamiliar humans (Bhattacharjee et al., 2017a). Interestingly, pet dogs have been shown to trust unfamiliar humans in a range of scenarios (see review Hare and Woods, 2013), which could possibly be the result of solely positive interspecific interactions. 
TABLE 3 | GLM results showing the effect of experimental conditions on gazing behavior in the FPP.

\begin{tabular}{lcccc}
\hline & Estimate & Standard error & z-value & $\operatorname{Pr}(>|z|)$ \\
\hline FIXED EFFECTS & & & & \\
Intercept & 1.1206 & 0.1043 & 10.748 & $<2 e-16^{\text {***}}$ \\
Condition FC & -1.8827 & 0.2869 & -6.563 & $5.28 \mathrm{e}-11^{\text {***}}$ \\
Condition HIT & 0.6315 & 0.1290 & 4.894 & $9.88 \mathrm{e}-07^{\text {***}}$ \\
Condition LIT & 0.4822 & 0.1326 & 3.636 & $0.000277^{\text {***}}$ \\
\hline
\end{tabular}

${ }^{\star \star \star} P \leq 0.001$

Nevertheless, pets are sensitive to behaviors of strangers (Vas et al., 2005), consistent with the results of this study.

Dogs' differential and situation-specific approach behaviors can be explained by early social interactions with humans (Fox and Stelzner, 1966). The role of domestication is also undeniable, which facilitated dogs' understanding and sensitivity toward human social cues. It has been shown that even hand-reared wolves (Canis lupus lupus), being the closest ancestors of modern day dogs failed to adjust behaviors while interacting with humans in ambiguous situations (Bradshaw and Nott, 1995). Thus, an interplay of domestication and factors like living environment and experience with humans might be the basis of the overall outcomes.

India has a huge population of both humans and free-ranging dogs, with regular interactions occurring between individuals of the two species, across habitats. More often than not, the outcomes of these interactions are adverse, leading to considerable dog-human conflict on the streets. This could be significantly reduced by having a more affiliative or at least, neutral approach toward free-ranging dogs instead of making them anxious or stressed. Studies on dog-human interactions on streets can help us to understand how human behavior can influence the behavior of the free-ranging dogs. This study reveals the effects of different social cues on the dogs, thus helping us to understand dogs' understanding and sensitivity to human social actions. One potential short-coming of the study was not being able to track individual dogs and failing to incorporate factors like frequency of positive and negative interactions with humans in their daily lives. Follow-up studies in different geographic regions with varying levels of human influences could be done to see the larger picture. It would also be interesting to test how free-ranging dogs respond to children, male and female experimenters with differing physical builds, while receiving

\section{REFERENCES}

Bertness, M. D., and Callaway, R. (1994). Positive interactions in communities. Trends Ecol. Evol. 9, 191-193. doi: 10.1016/0169-5347(94)90088-4

Bhadra, A., and Bhadra, A. (2014). Preference for meat is not innate in dogs. J. Ethol. 32, 15-22. doi: 10.1007/s10164-013-0388-7

Bhadra, A., Bhattacharjee, D., Paul, M., Singh, A., Gade, P. R., Shrestha, P., et al. (2016). The meat of the matter: a rule of thumb for scavenging dogs? Ethol. Ecol. Evol. 28, 427-440. doi: 10.1080/03949370.2015.1076526

Bhattacharjee, D., Nikhil Dev, N., Gupta, S., Sau, S., Sarkar, R., Biswas, A., et al. (2017a). Free-ranging dogs show age related plasticity in their ability to social cues from them. On the brighter side, this study has helped to identify a key element in the ecology of the dog-human relationship, the ability of the dogs to assess a social cue (and thus intent) of unfamiliar humans, which explains why dogs are one of the most successful species in sharing the same niche with humans.

\section{ETHICS STATEMENT}

The study design did not violate the Animal Ethics regulations of the Government of India (Prevention of Cruelty to Animals Act 1960, Amendment 1982). The protocol for the experiment was approved by the IISER Kolkata Animal Ethics Committee, as part of a larger project sanctioned by the SERB (EMR/2016/000595).

\section{AUTHOR CONTRIBUTIONS}

$\mathrm{DB}$ and $\mathrm{AB}$ conceived and designed the study. $\mathrm{DB}$ and SS performed the field experiments as E1 and E2, respectively. DB coded the videos with a second naïve coder. DB analyzed the data. The initial draft was written by $\mathrm{DB}$ and later reviewed and edited by $A B$. AB supervised the work. All authors gave final approval for publication.

\section{FUNDING}

DB was supported by INSPIRE Fellowship, Department of Science and Technology (DST), Govt. of India. SS was supported by a fellowship from the project fellowship (Project No. EMR/2016/000595) from Science and Engineering Research Board (SERB). The study was supported by IISER Kolkata.

\section{ACKNOWLEDGMENTS}

The authors would also like to thank Ms. Sudeshna Chakraborty for coding a few videos and playing the role of a naïve coder. Authors would also like to thank Indian Institute of Science Education and Research Kolkata (IISER K) for providing infrastructural support.

\section{SUPPLEMENTARY MATERIAL}

The Supplementary Material for this article can be found online at: https://www.frontiersin.org/articles/10.3389/fevo. 2018.00232/full\#supplementary-material 
Dorey, N. R., Udell, M. A. R., and Wynne, C. D. L. (2010). When do domestic dogs, Canis familiaris, start to understand human pointing? The role of ontogeny in the development of interspecies communication. Anim. Behav. 79, 37-41. doi: 10.1016/j.anbehav.2009.09.032

Fox, M. W., and Stelzner, D. (1966). Approach/withdrawal variables in the development of social behaviour in the dog. Anim. Behav. 14, 362-366. doi: 10.1016/S0003-3472(66)80098-2

Hare, B., Brown, M., Williamson, C., and Tomasello, M. (2002). The domestication of social cognition in dogs. Science 298, 1634-1636. doi: $10.1126 /$ science. 1072702

Hare, B., and Tomasello, M. (2005). Human-like social skills in dogs? Trends Cogn. Sci. 9, 439-444. doi: 10.1016/j.tics.2005.07.003

Hare, B., and Woods, V. (2013). The Genius of Dogs: Discovering the Unique Intelligence of Man's Best Friend. Dutton, MT: First NY. Available online at: https://books.google.co.in/books?hl=en\&lr=\&id=tFEjglZ5etYC\&oi= fnd\&pg=PR9\&dq=420\#v=onepage\&q\&f=false

Hughes, J., and Macdonald, D. W. (2013). A review of the interactions between free-roaming domestic dogs and wildlife. Biol. Conserv. 157, 341-351. doi: 10.1016/j.biocon.2012.07.005

Larson, G., Karlsson, E. K., Perri, A., Webster, M. T., Ho, S. Y., Peters, J., et al. (2012). Rethinking dog domestication by integrating genetics, archeology, and biogeography. Proc. Natl. Acad. Sci. U.S.A. 109, 8878-8883. doi: 10.1073/pnas.1203005109

Lindsay, S. R. (2005). Handbook of Applied Dog Behavior and Training, Volume Three, ed S. R. Lindsay Ames (Iowa, LA: Blackwell Publishing Professional), 122-167.

Miklósi, A., Polgárdi, R., Topál, J., and Csányi, V. (2000). Intentional behaviour in dog-human communication: an experimental analysis of "showing" behaviour in the dog. Anim. Cogn. 3, 159-166. doi: 10.1007/s100710000072

Miklósi, A., and Soproni, K. (2006). A comparative analysis of animals' understanding of the human pointing gesture. Anim. Cogn. 9, 81-93. doi: 10.1007/s10071-005-0008-1

Morey, D. F. (2006). Burying key evidence: the social bond between dogs and people. J. Archaeol. Sci. 33, 158-175. doi: 10.1016/j.jas.2005.07.009

Nagasawa, M., Murai, K., Mogi, K., and Kikusui, T. (2011). Dogs can discriminate human smiling faces from blank expressions. Anim. Cogn. 14, 525-533. doi: 10.1007/s10071-011-0386-5

Overall, K. L. (2014). The ethogram project. J. Vet. Behav. Clin. Appl. Res. 9, 1-5. doi: 10.1016/j.jveb.2013.12.001
Paul, M., Sen Majumder, S., Sau, S., Nandi, A. K., and Bhadra, A. (2016). High early life mortality in free-ranging dogs is largely influenced by humans. Sci. Rep. 6:19641. doi: 10.1038/srep 19641

Perri, A. (2016). A wolf in dog's clothing: Initial dog domestication and Pleistocene wolf variation. J. Archaeol. Sci. 68, 1-4. doi: 10.1016/j.jas.2016.02.003

R Development Core Team (2015). R: A language and environment for statistical computing. Vienna: R Foundation for Statistical Computing. Available online at: http://www.R-project.org/

Rehn, T., McGowan, R. T., and Keeling, L. J. (2013). Evaluating the strange situation procedure (SSP) to assess the bond between dogs and humans. PLoS ONE 8:e56938. doi: 10.1371/journal.pone.0056938

Sen Majumder, S., Bhadra, A., Ghosh, A., Mitra, S., Bhattacharjee, D., Chatterjee, J., et al. (2014). To be or not to be social: foraging associations of free-ranging dogs in an urban ecosystem. Acta Ethol. 17, 1-8. doi: 10.1007/s10211-013-0158-0

Takaoka, A., Maeda, T., Hori, Y., and Fujita, K. (2015). Do dogs follow behavioral cues from an unreliable human? Anim. Cogn. 18, 475-483. doi: 10.1007/s10071-014-0816-2

Thompson, J. N. (1999). The evolution of species interactions. Science 284, 2116-2118. doi: 10.1126/science.284.5423.2116

Vas, J., Topál, J., Gácsi, M., Miklósi, Á., and Csányi, V. (2005). A friend or an enemy? Dogs' reaction to an unfamiliar person showing behavioural cues of threat and friendliness at different times. Appl. Anim. Behav. Sci. 94, 99-115. doi: 10.1016/j.applanim.2005.02.001

Wynne, C. D. L., Udell, M. A. R., and Lord, K. A. (2008). Ontogeny's impacts on human-dog communication. Anim. Behav. 76, e1-e4. doi: 10.1016/j.anbehav.2008.03.010

Conflict of Interest Statement: The authors declare that the research was conducted in the absence of any commercial or financial relationships that could be construed as a potential conflict of interest.

Copyright (c) 2018 Bhattacharjee, Sau and Bhadra. This is an open-access article distributed under the terms of the Creative Commons Attribution License (CC BY). The use, distribution or reproduction in other forums is permitted, provided the original author(s) and the copyright owner(s) are credited and that the original publication in this journal is cited, in accordance with accepted academic practice. No use, distribution or reproduction is permitted which does not comply with these terms. 